\title{
Investigating Bio-interface Effects of Chronic ELF-MF Exposure before and after Neonatal Life on Rat Offspring Using Spectroscopy and Biochemical Assays
}

\author{
Zozan Guleken $^{1, *(D)}$, Devrim Sarıbal ${ }^{2}$ (D) , Cağlar Uyulan ${ }^{3}$ (D) , Aybike Keles ${ }^{4}$ (D) , Joanna Depciuch 5,* (D)
}

1 Department of Physiology, Uskudar University Faculty of Medicine, Istanbul, Turkey; zozangulleken@ gmail.com (Z.G.);

2 Department of Biophysics, Istanbul University Cerrahpaşa Faculty of Medicine, Istanbul, Turkey; devrimsaribal@gmail.com (D.S.);

3 Department of Mechatronics Engineering, Bulent Ecevit University, Zonguldak, Turkey; caglaruyulan@beun.edu.tr (C.U.);

4 Undergraduate student, Uskudar University Faculty of Health Sciences, Istanbul, Turkey; aybike.keles@ st.uskudar.edu.tr (A.K.);

5 Institute of Nuclear Physics Polish Academy of Science, 31-342 Krakow, Poland; joanna.depciuch@ifj.edu.pl (J.D.);

* Correspondence: zozanguleken@ gmail.mail.com (Z.G.); joanna.depciuch@ifj.edu.pl (J.D.);

Scopus Author ID 57118324800 (J.D.)

Scopus Author ID 57201342051 (Z.G.)

Received: 24.02.2021; Revised: 2.04.2021; Accepted: 5.04.2021; Published: 26.04.2021

Abstract: Electromagnetic (EM) energy that generates extremely low-frequency magnetic fields (ELFMF) impacts all living organisms' biological systems. To investigate the chronic impact of ELF-MF (50 $\mathrm{Hz})$ we designed an externally applied electric field exposure to rat offspring during intrauterine and after extrauterine life. A total of 18 rat offsprings blood serum was analyzed via biochemical assays and ATR-FTIR (400 to $4000 \mathrm{~cm}^{-1}$ ) analysis. The changes in the samples' total antioxidants (TAS) and total oxidants level (TOS) were measured, and we calculated oxidative stress index (OSI) and TNF- $\alpha$ level in serum samples. To attribute diseases, chemometric models were validated using PCA-SVD analysis. Proteins, including the amide $\mathrm{I}\left(1631 \mathrm{~cm}^{-1}\right)$, exhibit the strongest bands in the spectra depending on the secondary structure of the protein, and there was a degradation of lipids as a result of lipid peroxidation; also a significant increase in the lipid to protein ratio was concluded from the analysis $(\mathrm{p}<0.001)$. Finally, EMF exposure statistically significantly interferes with lipids, protein, and DNA/RNA molecules. Our findings demonstrate that chronic EMF exposure is an environmental factor affecting blood serum parameters and could impair oxidant-antioxidant function, increase lipid peroxidation and oxidative stress depending on the continuity of ELF-MF exposure.

Keywords: ELF-MF; prenatal exposure; postnatal exposure; blood serum; biochemical analysis; FTIR spectroscopy; biointerface.

\footnotetext{
(C) 2021 by the authors. This article is an open-access article distributed under the terms and conditions of the Creative Commons Attribution (CC BY) license (https://creativecommons.org/licenses/by/4.0/).
}

\section{Introduction}

Extremely low-frequency electromagnetic fields (ELF-EMF) are very low-energy radiation that interacts with biomolecular systems. This interaction has mostly initiated damage via reactive oxygen species (ROS), which can generate free radicals and early biological responses in the cellular environment such as DNA, lipids, and proteins. ROS involvement in ELF-MF exposure and various diseases and traceability and influence on the radical couple recombination has been documented in the past [1]. When ROS attacks lipids, a lipid peroxidation chain reaction starts. Lipid peroxidation is a key impact of electromagnetic fields 
on biological membranes. Peroxidation of membrane lipids may induce changes in the structure, permeability, and fluidity of membranes and cause many hazardous effects on their function. Affecting the antioxidant/prooxidant balance and reducing the antioxidant capacity, ROS were known to disturb the cellular microenvironment and modify normal cells' function [2]. Healthy embryonic development in fetal life is essential for the procreation of a population. Disorders caused by oxygen free radicals, which play an important role in the onset of many diseases during the neonatal period, may induce many pathological conditions after birth and postnatal period. Researches have shown that mammalians have regulated embryo-fetal development; that is, embryonic stem cells are strictly induced and modulated by signaling molecules during differentiation and the entire developmental process [3]. The neonate's adaptive immune system is not completely incapable but inexperienced and has no immunological memory due to the lack of in utero antigenic stimulation. Animal studies had been shown that exposure to EMF exposure did significant risk impaired implantation or the fetal development of animals. This is attributed to the cellular process involving mitosis and meiosis, which is the period of cells exposed to external stressors, like heat, physical and chemical agents [4].

The blood cells and components sensitively and effectively respond to internal stress factors such as bacteria, intracellular parasites, viruses, and external stress factors, including nutritional factors, toxins, alcohol, foreign bodies, and EMF exposure [5].

Rats exposed to EMF show increases in blood pressure, the whole heart, and left ventricular weights. The movement of hemoglobin in blood vessels is accelerated due to the presence of ferric ions. Also, exposure to EMFs causes structural differences in blood parameters such as a decrease in total antioxidant capacity and plasma calcium level, which are diagnostic for certain diseases such as anemia, leukemia, and inflammation of the human body. Studies had been shown that prolonged exposure to ELF-MF causes an increase in red blood cells (RBCs) count, decrease in white blood cell (WBCs) count and lymphocyte count, which leads to deterioration in large molecules in cells and imbalance in ionic equilibrium [6].

Generally, studies are focused on epidemiologic studies. These studies have a conclusion on MF exposure establish a link between human adverse effects on cell proliferation. Animal studies had been shown that exposure to ELF-MF did significant risk impaired implantation or fetal development in animal studies. This is attributed to the cellular process involving mitosis and meiosis, which is the period of cells exposed to external stressors, like heat, physical and chemical agents, MF exposure in the intrauterine period and after the developmental stage of living organism [7]. Although industrial agencies direct assurances of safety to avoid non-voluntary exposure, it seems unlikely that get rid of exposure completely is very hard.

However, long term and chronic effects of MFs are inconclusive, and there is not enough study about the relationship between chronic exposure to ELF-MF in the intrauterine and extrauterine period; studies had been showing that bimolecular interaction results in an imbalance in cellular progress, causing apoptosis on cell proliferation [8]. Nowadays, we are constantly exposed to electromagnetic fields.

Infrared (IR) spectroscopy is a powerful tool for biological tissues and biofluids such as whole blood, urine, saliva, and serum [9]. Classifying spectral categories lets us distinguish different categories in some clinical settings and the analyzed sample's underlying health status [10]. To understand probable structural and functional changes of biological molecules, as in 
previous studies, we used spectroscopic peak positions, band areas, and the band ratios of the samples, including the changes induced by ELF-MF exposure in biological systems [11-12].

There is no information about how exposure to a magnetic field during pregnancy affects offspring's blood serum level. Therefore, we designed an experimental set-up to evaluate juvenile rats' blood biochemical values before and after chronic magnetic field exposure. We used specific computational analysis techniques to obtain meaningful and reliable information to process IR spectra known as chemometrics. Also, we used preprocessing techniques to correct baseline and to remove not related possible variations, and then chemometric models were validated to obtain possible spectral biomarkers with sensitivity and specificity. As a multivariate classification model, we used principal component analysis plus singular value decomposition analysis (PCA-SVD). To see the oxidative status and immune response, we measured TAS, TOS, OSI and TNF- $\alpha$ levels.

\section{Materials and Methods}

\subsection{Animals and experimental design.}

Experimental protocols were approved by the Bezmialem University Animal Care and Use Committee (2020/95) in accordance with the Declaration of Helsinki and with the guidelines of the care and use of laboratory animals. Sprague Dawley rats (250-200 g), obtained from Bezmialem University Experimental Animals Research and Implementation Centre. They were housed in the standard cages one by one, under controlled environmental conditions with an ambient temperature of $\left(22 \pm 2^{\circ} \mathrm{C}\right)$ a 12-h light/dark cycles, and $60 \%$ humidity, during the experiment (lights on from 6:00 AM to 6:00 PM). Rats were nourished with standard rat chow and able to access water ad libitum.

At the beginning of the study, female Sprague-Dawley rats were mated with a cage design of two females per male. Pregnancy was determined by a vaginal smear test, and the detection of the sperm was designated as day 0 of pregnancy. Pregnant rats were removed and kept in separate cages. Pregnancy female rats weighed $(n=2) 250-300$ g were randomly distributed into two main groups: Exposed group placed into $50 \mathrm{~Hz} 500 \mu \mathrm{T}$ ELF-MF radiating magnetic field cages for 24 hours for the duration of pregnancy (21 days) for intrauterine (IU) period from day 0 of gestation to day 21 of lactation, while pregnant rats of the Unexposed group were placed in an inactive system for the same time. Next, the birth, newborn rats were also randomly distributed into two main groups: exposed to the same magnetic group $n=11$ and unexposed group $n=7$ group: conditions as during the IU period. The exposure time was 42 days to investigate the influence of the long-term and chronic impact of exposure on the magnetic field. After the $42^{\text {th }}$ days, newborn rats were anesthetized and sacrificed with ketamine (35 mg/kg, i.m.), and xylazine (5-10 mg/kg, i.m.) body trunk blood was collected by cardiac puncture for the measurement of blood IR spectroscopy analysis.

\subsection{ELF-MF exposure.}

Merritt coil system [13] was used as the source of ELF-MF. Merritt coil system, which can produce a large volume of the uniform magnetic field, consists of 4 square coils; inner coils have 11 windings, and outer coils have 26 windings. The system has 3 shelves, each 90x90cm in size, and 3 cages can fit on each shelf. In the trial studies conducted before and during our experiment, the uniform magnetic field intensity created by the cages was followed by averages of 6 minutes with Narda EHD-50D electric-magnetic field probe. The average magnetic field 
intensity on the shelves was determined as $500 \pm 8 \mu \mathrm{T}$ when the system was operating, and the magnetic field intensity of the room was determined as 10-12 $\mu \mathrm{T}$ when the lights were on in the background measurements. There were no other devices that may generate magnetic fields in the room where the coil system was located during the experiments. Faraday cage, which is used to block electric fields, did not need to be used in the experiment because it cannot block low-frequency magnetic fields such as ELF-MF. In order to obtain the required magnetic field strength, the variac was adjusted to give a constant electrical current of $13.39 \mathrm{~A}$ from enamel coated AWG $5(\mathrm{R}=4.62 \mathrm{~mm})$ thick single-core copper wire. No significant temperature increase was detected in the wires or in the environment when the system was operating. The constant relative humidity was measured as $50 \pm 10 \%$ and the temperature $24 \pm 1^{\circ} \mathrm{C}$ on the shelves with cages in the environment during the radiation.

ELF-MF was produced using the Merritt coil system, which produces $50 \mathrm{~Hz}$ frequency and $500 \mu \mathrm{T}$ field surrounded in a frame. The Merritt coil system consisted of 4 square coils; the inner coils 11 have windings and the outer coils 26 have windings, and the square coils are connected in series. Each exposed rat was placed into separate cages, and the cages were located on the shelves of the ELF-MF producing system within the study period. During the exposure, the ELF-MF magnitude was measured every six minutes with a magnetic field probe (Narda EHD-50D, Germany). The flowchart of the study is presented in Figure 1.

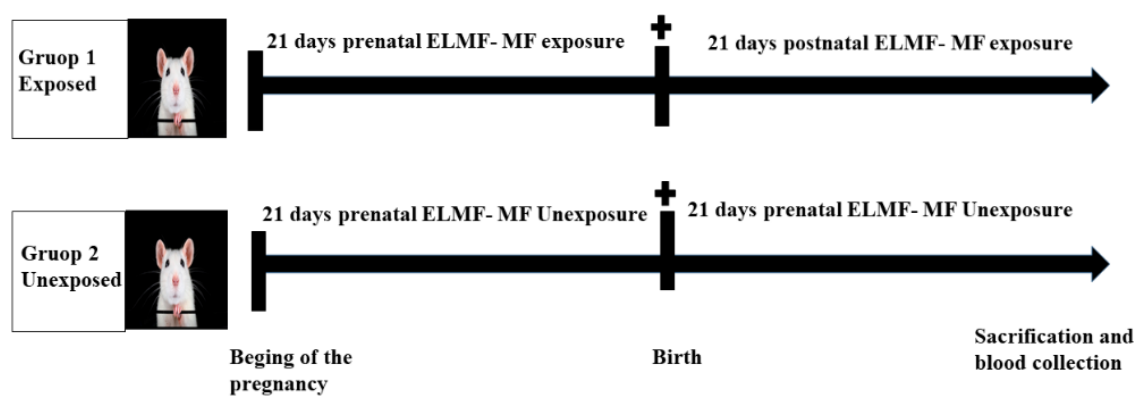

Figure 1. Flowchart of the experiment.

\subsection{Biochemical analyses.}

Total Antioxidant Status (TAS) levels were measured by the spectrophotometric method using a commercial kit (Rel Assay, Turkey). Thermo Scientific Microplate Reader (USA) was used to perform the assay. The changes in the samples' total antioxidants levels were measured at an absorbance of $660 \mathrm{~nm}$. TAS activities were expressed as mmol Trolox Equiv/L of samples. Total Oxidant Status (TOS) levels were measured by the spectrophotometric method using a commercial kit (Rel Assay, Turkey). Thermo Scientific Microplate Reader, USA device, was used to perform an assay. Results are indicated in terms of $\mu \mathrm{m} \mathrm{H}_{2} \mathrm{O}_{2}$ Equiv/L. Oxidative Stress Calculation (OSI): OSI) level for each patient calculated by using (TOS $(\mu$ mol. H2O2.equiv/L) $\times 100) /($ TAS $(\mu$ mol. Trolox.equiv/L) $)$ formula. TNF- $\alpha$ ELISA kit (Elabscience C., Cat no: E-EL-R0019, China) was used for the quantitative measurement of TNF- $\alpha$ in serum samples. Biotin was added to all wells and combined with Streptavidin-HRP to form an immune complex. Samples and standards were pre-coated with the Anti-Human monoclonal antibody before incubation, then carry out incubation again and washed to remove the uncombined enzyme. Optical density was read on a standard automated plate reader at $450 \mathrm{~nm}$ (Thermo Scientific Microplate Reader). The detection range of the kit was between 78.13-5000 pg/mL. 


\subsection{Spectral acquisition.}

Obtained blood samples from MF and Control rats were thawed at room temperature (20-30 minutes) for clotting, then $15 \mathrm{~min}$. centrifuged at $3000 \mathrm{rpm}$ for the isolation of the fibrinogen precipitate to obtain serum component. The obtained samples were stored at $-80^{\circ} \mathrm{C}$ until the analysis. We used JASCO FT/IR 4700 device Corporation 2967-5 Ishikawa-machi, Hachioji-shi, TOKYO, JAPAN) for spectroscopic measurements and the detection of the sample was made with liquid-nitrogen cooled mercury cadmium telluride (MCT). The device was containing a diamond with Gladi attenuated total reflection (ATR) TM, crystal plate, via appropriate software JASCO Spectra Manager version 2. IR spectral resolution was acquired at $4 \mathrm{~cm}^{-1}$ with zero-filling of the interferogram giving $4 \mathrm{~cm}^{-1}$ data spacing equivalent to 3684 data points with 32 scans within the $4000 \mathrm{~cm}^{-1}$ to $600 \mathrm{~cm}^{-1}$ regions. Before the measurement's samples were permitted to dehydrate at $24{ }^{\circ} \mathrm{C}$ in our temperature-controlled laboratory for approx. 5 minutes as previous studies [12]. Before each sample measurement, the empty diamond/MCT of ATR unit was recorded as background and repeatedly subtracted via the proper software to eliminate water in the sample and the air. To measure each sample spectrum, we transferred $1 \mu \mathrm{L}$ of blood serum onto an IR-reflective glass one by one to eliminate potential heterogeneity and waited for it to dry. Next, we acquired IR spectra of each sample from 20 difficult scenes for each sample for this sample. The crystal of the device was cleaned and dried before and after each sample's measurements. Two different experts have obtained measurements at different times. After the pre-processing, the selected spectral band areas were analyzed using the JWS set-up to detect the changes in animals' serum samples. Raw spectra were ATR from 4000 to $600 \mathrm{~cm}^{-1}$ spectral regions, baseline-corrected, vector normalized, 25 points smoothed with Savitzky-Golay to eliminate sharp bends in the spectral regions [13]. Moreover, ATR correction was applied. To obtain chemometric analysis, all features were averaged for serum sample spectral data.

\subsection{Computational analysis.}

2.5.1. Singular value decomposition (SVD) and principal component analysis (PCA).

We implemented the spectroscopic data of serum samples algorithms applications using Python sci-kit-learn library via Jupyter Notebook. The obtained serum samples from the animals were classified as ELF-MF exposed and unexposed groups. At this stage, we decomposed a numerically stable matrix to obtain related specific information about the samples' spectra. We used singular value decomposition (SVD) as a data-driven method that provides a low-dimensional reduction to high-dimensional data extracting for the dominant patterns. We used SVD because SVD is a numerically more generic, robust, and efficient machine learning method than other classifying methods [14]. PCA is a powerful analysis method to minimize high featured data with linear revolutions. The extracted features are named as principal components. PCA implements a pre-processing issue involving means subtraction and variance setting to unity before SVD. The resulting coordinate system's geometry is constituted by principal components that are uncorrelated (orthogonal) to each other but have a maximal correlation with the measurements. We used Python software for the spectral data between $4000 \mathrm{~cm}^{-1}$ to $600 \mathrm{~cm}^{-1}$. 


\subsection{Statistics.}

Kolmogorov-Smirnov was used to explore distribution. An Independent t-test, Oneway ANOVA (Graph Pad Prism 6.01 (Graph Pad Software, Inc.) was used to evaluate significance. We evaluated the absorbance of data as mean \pm SD (standard deviation). The statistical significance was signified as less than or equal to $\mathrm{p}<0.05^{*}, \mathrm{p}<0.01^{* *}$ and $\mathrm{p}<$ $0.001 * * *$.

\section{Results and Discussion}

In this study, long-term effects of EMF, starting with fetal life and continuing after birth, were investigated by FTIR spectroscopy and biochemical assays.

Sum levels of TAS, TOS, TNF- $\alpha$ were studied in Control and MF groups. We compared the levels of measurements with Mean \pm Std. Deviation, One-way ANOVA "', 'Bonferroni's multiple comparisons tests, the statistical results are shown in Table 1. As it is presented in Figure 2 our results showed significant changes among groups. There was a decrease in the serum levels of TAS in chronically exposed rats against the unexposed group ( $\mathrm{p}$-value $<0.05$ ). However, TOS measurements increased among chronically exposed rats ( $\mathrm{p}$-value < 0.05 ). Additionally, we compared the OSI index based on the ratio of reactive oxygen species (ROS) and antioxidant capacity among groups significantly higher in the exposed group. Also, serum levels of TNF- $\alpha$, which is almost used as a marker of cytokine in inflammation, were measured. The results showed a significant increase in the exposed group.

We showed FTIR spectra and second derivative of FTIR spectra collected from the blood serum of rats from MF and Control groups (Figure 3). When we compared the mean average FTIR spectral analysis results among these two experimental groups, we observed that in the control group, the value of absorbance in all IR ranges is lower than in MF group (Figure 4).

This maximum absorbance in the IR range corresponds to the characteristic's functional groups of nucleic acid, proteins, carbohydrates, and lipids are shown in Figure 5. Table 2 presents the vibrations assigned in the blood serum of samples and the description of these groups. There were significant quantitative changes in the nucleic acids (Figure 5a), proteins (Figure 5b), and lipids (Figure 5c). We observed a decrease of nucleic acids and phospholipids and an increase of proteins and lipids fraction in blood serum of rats' administration by EMF compared to the control group $(\mathrm{p}<0.001)$. Furthermore, one peak corresponding to proteins vibration was visible in this region. This peak corresponds to $\delta \mathrm{N}-\mathrm{H}$ bending, $\mathrm{C}-\mathrm{N}$, and $\mathrm{C}-\mathrm{C}$ stretching vibrations of amide III $-1234 \mathrm{~cm}^{-1}[15,16]$. Moreover, peaks at $1170 \mathrm{~cm}^{-1}$ attributed to $\mathrm{C}-\mathrm{H}$ tyrosine's in-plane bending mode [17] were observed. Furthermore, a peak at $1120 \mathrm{~cm}^{-}$ ${ }^{1}$ is attributed to the strong $\mathrm{C}-\mathrm{O}$ band of ribose (serves as a marker band for RNA in solutions) [18]. Moreover, peaks corresponding to nucleic acids and phospholipids vibrations were also noticed in measured samples. In FTIR spectra were visible peaks at $1078 \mathrm{~cm}^{-1}\left(\mathrm{C}-\mathrm{C}\right.$ or $\mathrm{PO}^{2}$ stretch (nucleic acid) [17] and $1029 \mathrm{~cm}^{-1}$ (asymmetric $\mathrm{PO}_{2}$ stretching vibration form phospholipids and nucleic acid) [19]. 



Figure 2. Levels of total antioxidant status (a) (TAS), total oxidant status TOS and oxidative stress index (OSI). TNF-alpha level for the exposed and unexposed group. ${ }^{*} \mathrm{P}<0.05$ and $* * * \mathrm{p}<0.001$ were significant among the exposed and unexposed groups.

Table 1. Plasma TAS, TOS, TNF- $\alpha$ and OSI (Oxidative stress index) level with Mean \pm Std. Deviation, Oneway ANOVA "', 'Bonferroni's multiple comparisons test results.

\begin{tabular}{l|c|c|c} 
Parameters & Unexposed & Exposed & p-value \\
\hline TAS & $0.79 \pm 0.063$ & $1.02 \pm 0.151$ & $\mathrm{p}<0.05$ \\
\hline TOS & $5.47 \pm 0.99$ & $5.712 \pm 0.227$ & $\mathrm{p}<0.05$ \\
\hline TNF- $\alpha$ & $864.6 \pm 202.3$ & $1018 \pm 65.67$ & $\mathrm{p}<0.001$ \\
\hline OSI & $5.21 \pm 1.51$ & $4.81 \pm 0.798$ & $\mathrm{p}<0.05$
\end{tabular}
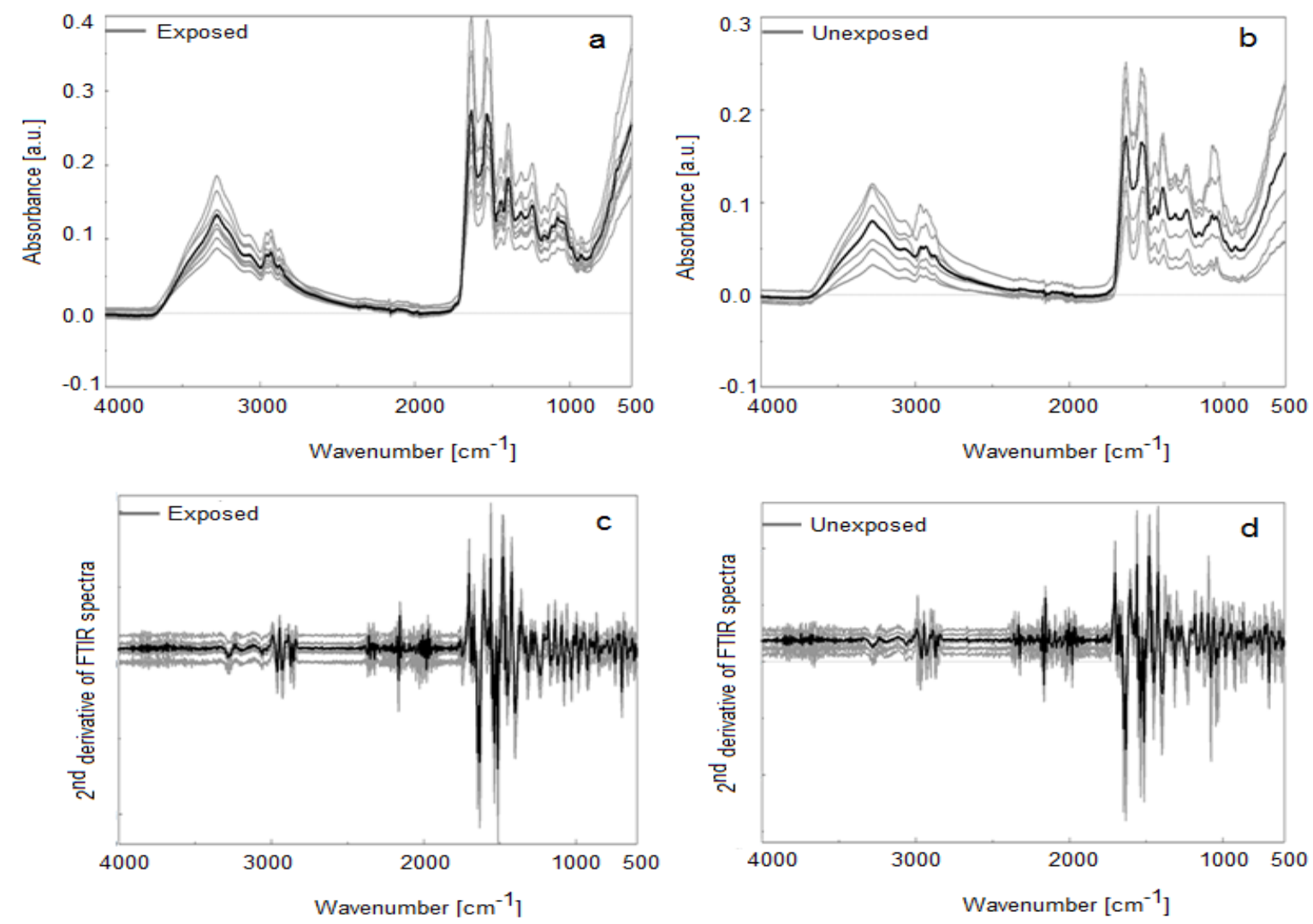

Figure 3. Raw FTIR spectra $(a, b)$ and second derivative of FTIR spectra $(c, d)$ of blood serum of newborn rats from MF (a, c) and Control (b, d) groups.

The characteristic IF proteins were observed (Figure $5 \mathrm{~b}$ ). The peak at $1693 \mathrm{~cm}^{-1}$ belongs mostly to the stretching of $\mathrm{C}=\mathrm{O}$ [20]. Proteins, including the amide $\mathrm{I}\left(1631 \mathrm{~cm}^{-1}\right)$, exhibit the spectra's strongest bands depending on the protein's secondary structure. Moreover, peaks at: $1535 \mathrm{~cm}^{-1}$ (Amide II) and $1513 \mathrm{~cm}^{-1}$ (cytosine) [21], were noticed in FTIR spectra.

The last analyzed region attributed to lipids vibrations was between $3000 \mathrm{~cm}^{-1}$ and 2800 $\mathrm{cm}^{-1}$ (Figure 5c). In these IR range vibrations corresponding to $\mathrm{CH}$ stretch of lipids and 
proteins, end asymmetric $\mathrm{CH}$ stretch band, $\mathrm{C}-\mathrm{H}$ vibrations in lipids \& proteins, vas $\mathrm{CH}_{2}$ [22], general assignments, methylene $\left(\mathrm{CH}_{2}\right), v_{s} \mathrm{CH}_{3}$, symmetric vibration of $\mathrm{CH}$ and general $\mathrm{CH}_{2}$, $\mathrm{CH}_{3}$ lipids vibrations [23-24], were observed.
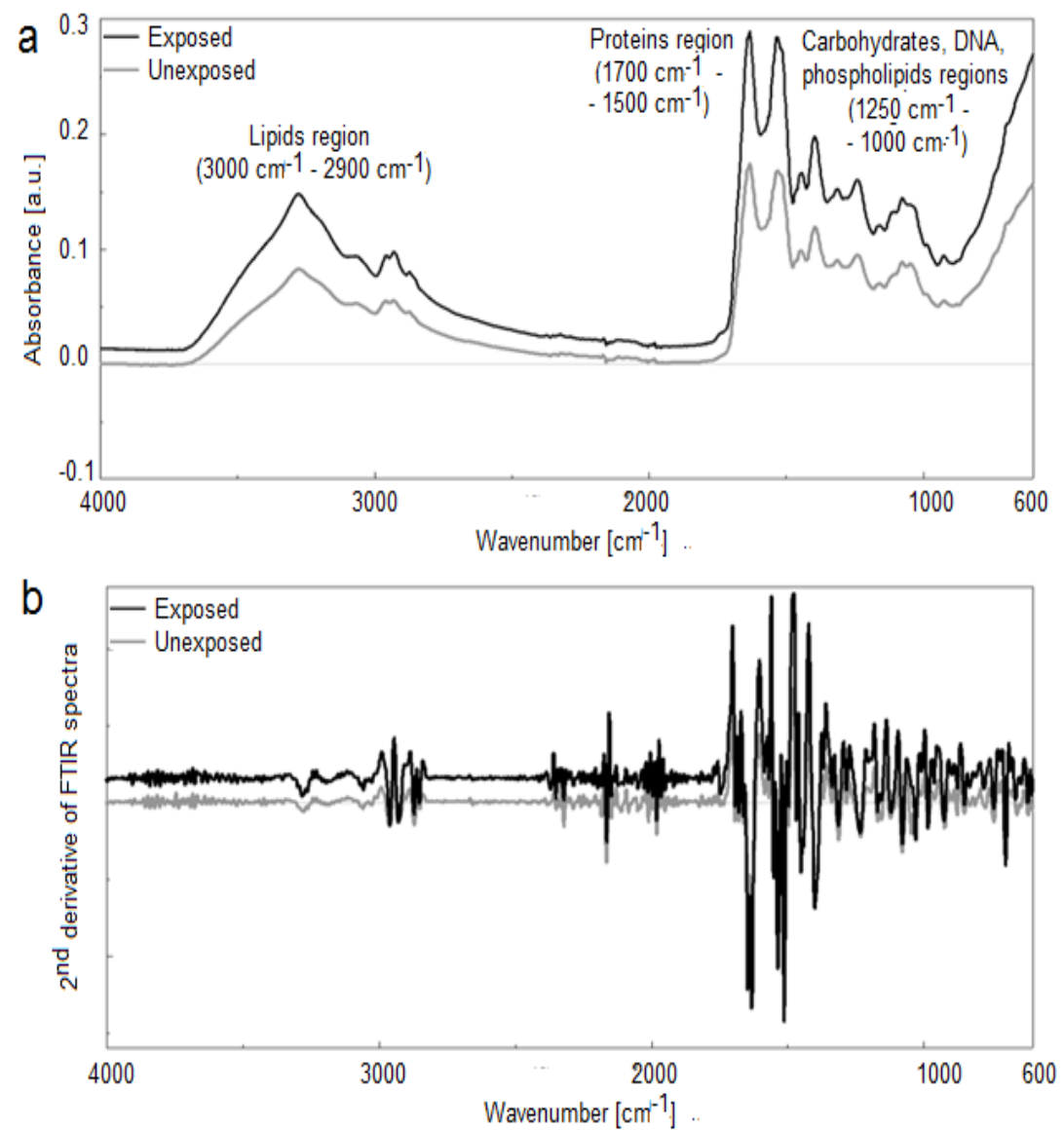

Figure 4. Mean FTIR spectra (a) and $2^{\text {nd }}$ derivative FTIR spectra (b) obtained for the blood serum of newborn rats from MF (black curve) and Control (grey curve) groups.
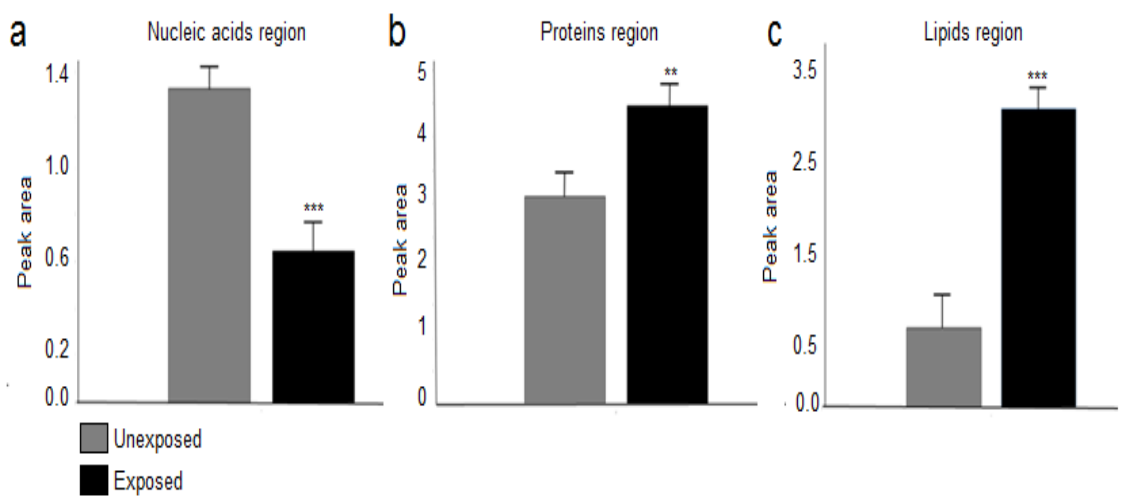

Figure 5. Band area ratio values of protein region $\left(1700 \mathrm{~cm}^{-1}-1500 \mathrm{~cm}^{-1}\right)\left(\right.$ a), lipid region $\left(3000 \mathrm{~cm}^{-1}-2800 \mathrm{~cm}^{-1}\right)$ (b) and nucleic acid region $\left(1250 \mathrm{~cm}^{-1}-1000 \mathrm{~cm}^{-1}\right)$ (c) for MF and Control group. The statistical significance was signified as less than or equal to $\mathrm{p}<0.01 * *$, $\mathrm{p}<0.001 * * *$.

Table 2. Spectral markers of IR absorption band assignments in blood serum.

\begin{tabular}{c|l|r}
$\begin{array}{c}\text { FTIR spectroscopy } \\
\text { Wavenumber }\left[\mathbf{c m}^{-1}\right]\end{array}$ & \multicolumn{1}{|c}{ Vibrations } & References \\
\hline 1234 & Amide III (proteins $\delta$ N-H bending, C-N, and C-C stretching) & {$[14]$} \\
\hline 1078 & C-C or PO2 stretch (nucleic acid) & {$[16]$} \\
\hline 1170 & C-H in-plane bending mode of tyrosine & {$[16]$} \\
\hline 1120 & The strong C-O band of ribose (serves as a marker band for RNA in solutions) & {$[17]$} \\
\hline 1029 & Asymetric PO2 stretching vibration form phospholipids and nucleic acid & {$[20]$} \\
\hline 1693 & Stretching of C=O & {$[21]$} \\
\hline 1631 & Amide I, & {$[22]$} \\
https://biointerfaceresearch.com/ & $\mathbf{8 0 2}$
\end{tabular}




\begin{tabular}{c|l|r}
$\begin{array}{c}\text { FTIR spectroscopy } \\
\text { Wavenumber }\left[\mathbf{c m}^{-1}\right]\end{array}$ & Vibrations & References \\
\hline 1513 & Cytosine & {$[24]$} \\
\hline 1535 & Amide II with lower absorbance found & {$[24]$} \\
\hline 2960 & End antisymmetric CH3 stretch band & {$[25]$} \\
\hline 2930 & General assignments, methylene (CH2) & {$[26]$} \\
\hline 2870 & Symmetric vibration of CH lipids group & {$[26]$}
\end{tabular}

We underwent the spectral data of chemometric analysis using PCA-SVM as a first discriminant attempt and then by PCA- k-means clustering analysis applied as a final and best discriminant model (Figure 6). We investigated exposed group serum samples and unexposed group serum samples. The size of the data matrices for the unexposed group's serum data was $7 \times 1765$, and 11x1765 for ELF-MF exposed groups obtained from the spectra of the samples. These data matrices represent absorbance corresponding to the wavenumbers. The length of the wavenumber vector was 1756 . The wavenumbers spanned from $4000 \mathrm{~cm}^{-1}$ to $600 \mathrm{~cm}^{-1}$. In this study, the PCA of the second derivative spectral data matrix standardized and K-means algorithm applied. For each of the 18 samples for serum configurations, one cluster analysis with $\mathrm{k}=2$ was performed (Figure 7). Also, Additionally, Table 3 shows the clustering evaluation metrics of plasma second derivative IR spectral data, where can be seen data distinguished successfully.

As it is known that the effect of chronic EMFs exposure does not fully know, therefore, we examined obtained blood serum via spectrochemical and biochemical analysis to evaluate the impact of chronic ELF-MF exposure in rats before and after neonatal life.

Our results showed that ELF-MF exposure affects the antioxidant system as mediators of metabolites of toxic effects via biochemical analysis, and spectral analysis showed that chronic EMF has the potential to change the balance of phospholipids, as well as lipids, carbohydrates, and proteins.


Figure 6. PCA analyses of blood serum obtained for raw FTIR data (a) and $2^{\text {nd }}$ derivative data (b) collected from MF (black dot) and Control (grey star) rats. The IR range was between 800 to $1800 \mathrm{~cm}^{-1}$. The size of the data matrices for serum data of the unexposed group was 7x1765, and 11x1765 for ELF-MF exposed groups obtained from the spectra of the samples. These data matrices represent absorbance corresponding to the wavenumbers. The length of the wavenumber vector was 1756 . The wavenumbers spanned from $4000 \mathrm{~cm}^{-1}$ to $600 \mathrm{~cm}^{-1}$.

In this study, the impact of chronic exposure increased animals' serum levels linked to an evoke on the antioxidant system. Torres-Duran et al. had been shown there was an increase in serum HDL after acute one-time exposure [25]. Increased lipoprotein peroxidation is a marker of modification of low-density lipoprotein [26]. ELF-MF exposure seems to have effects on the impaired antioxidant capacity. Additionally, increased levels of FFA in our study 
might indicate that metabolism of lipids was increased after ELF-MF exposure as in previous studies [25].

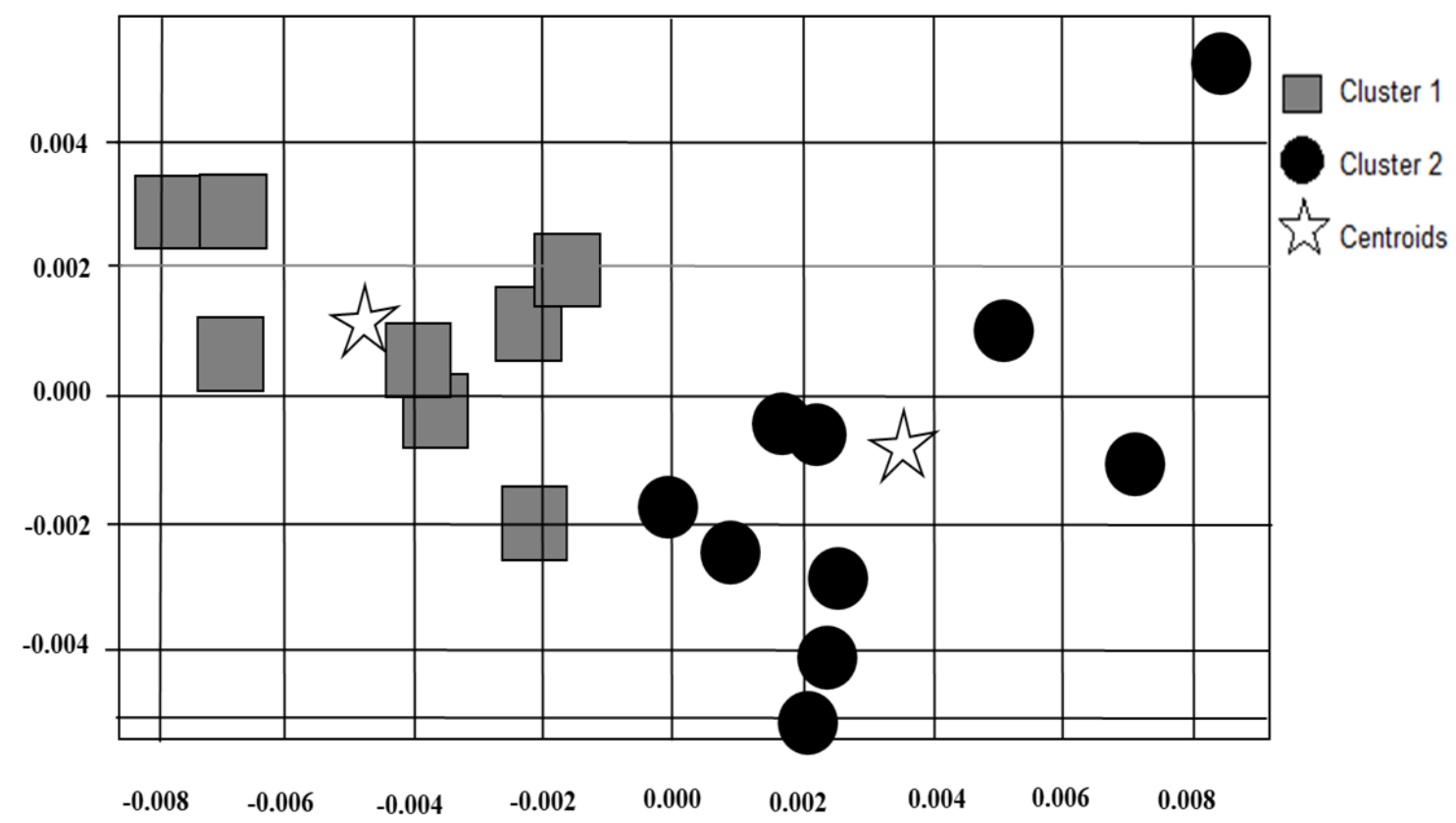

Figure 7. Cluster analyses of PCA result for $2^{\text {nd }}$ derivative data (C2->10, C1->8).

Serum TNF- $\alpha$ level was decreased in the chronic exposure group. This may be an interaction between TNF alpha and IL-1. As an inflammatory, stimulating cytokine, increased production IL-1 could increase oxidative stress, which might evoke inflammation. Contralaterally increased oxidative stress because of inflammation causes the production of ROS through inflammatory cells. Our results were following previous studies' findings [2728]. Lee et al. suggested that ELFMF exposure affects the antioxidant system by producing free ROS [29]. Additionally, some studies claimed that chronic ELF-MF exposure may lead to chronic diseases like atherosclerosis, damage to the liver, and brain regions changes. Finally, our biochemical analysis showed that chronic ELFMF exposure causes lipid peroxidation and antioxidant serum activity elevation and has impacts on membrane lipid balance.

Earlier studies had been showing that EMFs cumulative effects depend on the exposure time and intensity. EMFs' impact on animals showed that exposures over 1 or 2 months lead to modest structural changes on the brain and neurons, which may be irreversible. [30]. Also, Özorak et al. [31] showed that ELFs affect the reproductive system, which suggests structural changes and fertility problems leading to reproductive dysfunctions. These results suggest that EMF exposure shows its effect depending on cumulative and mutational effects. Consequently, in our results, we showed changes in the phospholipids and lipids IR regions (Figure 3-2, Figure 5a-c). These molecules mainly build brain structures [32]. Moreover, these changes are statistically significant and were observed after exposure of animals to a magnetic field (Figure 5a-c).

Exposing to the electrical field has significant effects on biological materials via changing electrical signals. Electrical signals have an important role in the transmission of information in the process of biological events. This interaction is sufficient for the critical biochemical changes of blood [33]. Membrane interaction with ELF-MF causes a change in the cell electrical signal order. Low frequency and low-intensity EM fields have effects on the movements of molecules and ions, which lead to improving the balance of ions in the body. 
Clinical evaluation of ELF-MF exposure resulted from the numerous changes of white blood cells and red blood cells. Moreover, in membrane receptors responsible for transmitting and receiving information from the outside were placed. These receptors are mainly made of protein structures. Therefore, the secondary structure of proteins is very important for their proper functioning. In our research, we showed that ELF-MF caused changes in protein fraction (Figure 3-4, Figure 5b) and the secondary structure of proteins (Figure 5b). Additionally, Ross et al. indicated that exposure to an ELF-MF less than $200-300 \mathrm{~Hz}$ might alter changes in cell metabolism resulting in the generation of free oxygen radicals causing oxidative stress [34], which also is responsible for changes in proteins.

ELF-MF originating from residential and industrial power lines, medical devices, and household appliances is a modern environmental pollution type produced by various electronic devices in everyday use. This repeated exposure results from various side effects on biological homeostasis, prompting several changes in biochemical blood levels to physiological diseases. Previous studies indicated that EMF affects cell cycles, cell viability, steroidogenesis [35] in the uterus, effects on metabolic activity. Besides, it affects the reproductive system, which changes the intrauterine milieu of estrogens in the endometrium tissue, leading to changes in the menstrual cycle and metabolism. Additionally, it has effective changes on membrane macromolecules and the metabolism of calcium. Our study showed that EMF changed the balance of phospholipids and lipids, carbohydrates, and proteins (Figure 5). Consequently, EMF caused changes in all organisms.

Recent studies about the effect of prenatal ELF-MF exposure showed that there are associations between low birth weight, small gestational age, or intrauterine growth retardation in offspring; also, human studies had shown that MF exposure might cause physical changes in humans. Yolk proteins, which provide nutrients to embryos, are essential for growth. It has large amounts of phosphor glycoproteins, amino acids, phosphate, lipids, and carbohydrates [36]. Additionally, it has a large number of mitochondria for the utilization of ATP production. An increase of glycogen functional groups was observed in the FTIR spectra (Figure 2) of the chronic EMF exposure group compared to the unexposed group. Our results were similar to a study that was made on the reproductive system [37].

As a result, EMF-MF is an important environmental factor in developing cells that have a fast-mitotic activity. Our study verified that chronic low-frequency magnetic field exposure affects rats' pups' blood serum structure exposed in fetal life and after birth. IR analysis had provided information about chemical and functional vibrations underexposure or unexposed environmental state. We used the IR spectroscopic method because of these methods' advantages in recent studies to evaluate blood serum biological samples.

The research outputs clarify that long-term ELF- MF exposure to rats' pups before and after gestational period basis appreciable long-term deficit in blood parameters. Our findings demonstrate that chronic $50 \mathrm{~Hz}$ exposure impacts lipid profile, increases lipid peroxidation, and affects the antioxidant system. Also, we concluded that FTIR might be a selectable technique that can be used to assess the effects of EMF on the blood tissue of animals. However, recent literature does not have enough knowledge about the interaction of ELF- MF and biological material. There is a big necessity to understand the interaction and biological systems; for this reason, we need further research and analysis. 


\section{Conclusions}

Considering that the use of EMFs with several spectrums involved in the natural environment will increase even more in the future, the subject's importance increases. However, there is not enough study about intrauterine and extrauterine exposure to the low frequency of magnetic field and biomolecular interaction and chronic impact of ELF-MF exposure on blood tissue physiology. Our results showed that long-term exposure to ELF-MF caused quantitative and qualitative changes in phospholipids, nucleic acid, carbohydrates, proteins, and lipids. Moreover, obtained FTIR spectra showed decreased phospholipids fraction and increased proteins and lipids amount in blood serum. Furthermore, significant changes in proteins were visible in rats that have been subjected to a magnetic field. Consequently, this study showed that ELF -MF exposure caused significant changes in biological molecule balance in blood serum, which may have consequences for the body's proper functioning. However, these effects should be investigated in the following study.

\section{Funding}

This research received no external funding.

\section{Acknowledgments}

This research has no acknowledgment.

\section{Conflicts of Interest}

The authors declare no conflict of interest.

\section{References}

1. Merla, C.; Liberti, M.; Consales, C.;Denzi, A.; Apollonio, F.; Marino, C.; Benassi, B. Evidences of plasma membrane-mediated ROS generation upon ELF exposure in neuroblastoma cells supported by a computational multiscale approach. Biochimica et Biophysica Acta-Biomembranes 2019, 1861, 1446-1457, https://doi.org/10.1016/j.bbamem.2019.06.005.

2. Güleken, Z. Chronic low-frequency electromagnetic field exposure before and after neonatal life induces changes on blood oxidative parameters of rat offspring. Annals of Medical Research 2021, 28, 361-5, https://doi.org/10.5455/annalsmedres.2020.08.876.

3. Kowalczuk, C.I.; Lucy R.; Thomas, J.M. Effects of Prenatal Exposure to $50 \mathrm{~Hz}$ Magnetic Fields on Development in Mice: I. Implantation Rate and Fetal Development. Bioelectromagnetics 1994, 15, 349-61, https://doi.org/10.1002/bem.2250150409.

4. Núñez-Enríquez, J.C.; Correa-Correa, V.; Flores-Lujano, J; Pérez-Saldivar, M.L.; Jiménez-Hernández, E; Martín-Trejo, J.A; Espinoza-Hernández, L.E.; Medina-Sanson, A.; Cárdenas-Cardos, R; Flores-Villegas, L.V. Extremely Low-Frequency Magnetic Fields and the Risk of Childhood B-Lineage Acute Lymphoblastic Leukemia in a City With High Incidence of Leukemia and Elevated Exposure to ELF Magnetic Fields. Bioelectromagnetics 2020, 41, 581-597, https://doi.org/10.1002/bem.22295.

5. Khan, M.W.; Juutilainen, J.; Auvinen, A.; Naarala, J.; Pukkala, E.; Roivainen, P. A cohort study on adult hematological malignancies and brain tumors in relation to magnetic fields from indoor transformer stations. International Journal of Hygiene and Environmental Health 2021, 233, https://doi.org/10.1016/j.ijheh.2021.113712.

6. Ren, Y.; Chen, J.; Miao, M.; Li, D.K.; Liang H.; Wang, Z.; Yang, F.; Sun, X.; Yuan, W. Prenatal exposure to extremely low frequency magnetic field and its impact on fetal growth. Environmental Health 2019, 18, https://doi.org/10.1186/s12940-019-0447-9.

7. Zastko, L.; Makinistian, L; Moravčíková, A; Jakuš, J.B.I. Effect of Intermittent ELF MF on Umbilical Cord Blood Lymphocytes. Bioelectromagnetics 2020, 41, 649-655, https://doi.org/10.1002/bem.22302.

8. Chen, L.; Xia, Y.; Lu, J.; Xie, Q.; Ye, A.; Sun, W. A 50-Hz magnetic-field exposure promotes human amniotic cells proliferation via SphK-S1P-S1PR cascade mediated ERK signaling pathway. Ecotoxicology and Environmental Safety 2020, 194, https://doi.org/10.1016/j.ecoenv.2020.110407. 
9. Güleken, Z.; Ünübol, B.; Toraman, S. Diagnosis of Opioid Use Disorder with High Sensitivity and Specificity by Advanced Computational Analysis of Fourier Transform Infrared Spectroscopy. Infrared Physics and Technology 2020, 105, https://doi.org/10.1016/j.infrared.2020.103218.

10. Guleken, Z.; Ünübol, B.; Bilici, R. Investigation of the Discrimination and Characterization of Blood Serum Structure in Patients with Opioid Use Disorder Using IR Spectroscopy and PCA-LDA Analysis. journal of pharmaceutical and biomedical analysis 2020, 190, https://doi.org/10.1016/j.jpba.2020.113553.

11. Morais, C.L.M.; Paraskevaidi, M.; Cui, L. Standardization of Complex Biologically Derived Spectrochemical Datasets. Nature Protocols 2019, 14, 1546-77, https://doi.org/10.1038/s41596-019-0150$\mathrm{X}$.

12. Guleken, Z.; Kuruca, S.E.; Ünübol, B. Biochemical Assay and Spectroscopic Analysis of Oxidative/Antioxidative Parameters in the Blood and Serum of Substance Use Disorders Patients. A Methodological Comparison Study. Spectrochim Acta A Mol Biomolecular Spectroscsopy 2020, 240, https://doi.org/10.1016/j.saa.2020.118625.

13. Merritt, I.R; Purcell, C; Stroink, G. Uniform magnetic field produced by three, four, and five square coils, Review of Scientific Instruments 1983, 54, 879-882, doi.org/10.1063/1.1137480

14. Depciuch, J.; Parlinska-Wojtan, M. Qualitative and Quantitative Changes in Phospholipids and Proteins Investigated by Spectroscopic Techniques in Olfactory Bulbectomy Animal Depression Model. Journal of Pharmaceutical and Biomedical Analysis 2018, 148, 24-31, https://doi.org/10.1016/j.jpba.2017.09.016.

15. Steck, T.L.; Kant, J.A. Preparation of Impermeable Ghosts and inside-out Vesicles from Human Erythrocyte Membranes. In Methods in Enzymology. Methods Enzymology 1974, 31, 172-80, https://doi.org/10.1016/0076-6879(74)31019-1.

16. Keser, H.; Bozkurt, Girit Ö.; Majeed, M.; Nayak, M.; Bilgin, M.D. Pterostilbene administration improves the recovery potential of extremely low-frequency magnetic field in acute renal ischemia-reperfusion injury: an FTIR spectroscopic study. Turkish Journal of Biology 2020, 17, 48-60, https://doi.org/10.3906/biy-190718.

17. Steinier, J.; Termonia, Y.; Deltour, J. Smoothing and Differentiation of Data by Simplified Least Square Procedure. Analytical Chemistry 1972, 44, 1906-9, https://doi.org/10.1021/ac60319a045.

18. Singh P. Supervised Machine Learning. In: Learn PySpark: Build Python-Based Machine Learning and Deep Learning Models. edited by Pramod, S.B. CA: Apress 2019; pp. 117-59, https://doi.org/10.1007/9781-4842-4961-1_6.

19. Henry, D.; Allison, B.D.; Nathaniel, M. Clustering Methods with Qualitative Data: A Mixed Methods Approach for Prevention Research with Small Samples. Prevention Science: The Official Journal of the Society for Prevention Research 2015, 16, 1007-16, https://doi.org/10.1007/s11121-015-0561-z.

20. Chaber, R.; Lach, K.; Depciuch, J. Fourier Transform Infrared (FTIR) Spectroscopy of Paraffin and Deparafinnized Bone Tissue Samples as a Diagnostic Tool for Ewing Sarcoma of Bones. Infrared Physics \& Technology 2017, 85, https://doi.org/10.1016/j.infrared.2017.07.017.

21. Callery, E.L; Morais, C.L.M.; Paraskevaidi, M. New Approach to Investigate Common Variable Immunodeficiency Patients Using Spectrochemical Analysis of Blood. Scientific Reports 2019, 9, https://doi.org/10.1038/s41598-019-43196-5.

22. Diem, M; Chalmers, J.M.; Griffiths, P.R. Vibrational Spectroscopy for Medical Diagnosis. John Wiley \& Sons: Chichester, U.K.; Hoboken, N.J. USA. 2008,

23. Ruiz-Chica, A.J.; Medina, M.A.; Sánchez-Jiménez, F. Characterization by Raman spectroscopy of conformational changes on guanine-cytosine and adenine-thymine oligonucleotides induced by aminooxy analogues of spermidine, Journal of Raman Spectroscopy 2004, 35, 93-100, https://doi.org/10.1002/jrs.1107.

24. Sigurdsson, S.; Philipsen, P.A.; Hansen, L.K. Detection of skin cancer by classification of Raman spectra. IEEE Trans Biomedical Engineering 2004, 51, 1784-1793, https://doi.org/10.1109/tbme.2004.831538.

25. Torres-Duran, P.V.; Ferreira-Hermosillo, A. Effects of Whole-Body Exposure to Extremely Low Frequency Electromagnetic Fields (ELF-EMF) on Serum and Liver Lipid Levels, in the Rat'. Lipids and Health Disease 2007, 16, 6-31, https://doi.org/10.1186/1476-511X-6-31.

26. Gutteridge, J.M.; Halliwell, B. The Measurement and Mechanism of Lipid Peroxidation in Biological Systems. Trends in Biochemical Sciences 1990, 15, 129-35, https://doi.org/10.1016/0968-0004(90)90206q.

27. Akdag, M.; Dasdag, Z.S.; Ulukaya, E.; Uzunlar, A.K. Effects of Extremely Low-Frequency Magnetic Field on Caspase Activities and Oxidative Stress Values in Rat Brain. Biological Trace Element Research 2010, 138, 238-49, https://doi.org/10.1007/s12011-010-8615-3.

28. Zwirska-Korczala, K.; Jochem, J.; Adamczyk-Sowa, M. Effect of extremely low frequency electromagnetic fields on cell proliferation, antioxidative enzyme activities and lipid peroxidation in 3T3-L1 preadipocytes An in vitro study. Journal of Physiology and Pharmacology 2005, 101-108.

29. Hanie M, Jabarivasal; N, Sardanian, K. Effects of Various Densities of $50 \mathrm{~Hz}$ Electromagnetic Field on Serum IL-9, IL-10, and TNF- $\alpha$ Levels. International Journal of Occupational and Environmental Medicine 2020, 11, 24-32, https://doi.org/10.15171/ijoem.2020.1572. 
30. Le-Deygen, I.M.; Vlasova, K.Y.; Kutsenok, E.O.; Usvaliev, A.D.; Efremova, M.V.; Zhigachev, A.O.; Rudakovskaya, P.G.; Golovin, D.Y.; Gribanovsky, S.L.; Kudryashova, E.V. Magnetic nanorods for remote disruption of lipid membranes by non-heating low frequency magnetic field. Nanomedicine 2019, 21, https://doi.org/10.1016/j.nano.2019.102065.

31. Ozorak, A; Nazıroğlu, M; Celik, O. Wi-Fi (2.45 GHz)- and Mobile Phone (900 and $1800 \mathrm{MHz}$ )-Induced Risks on Oxidative Stress and Elements in Kidney and Testis of Rats During Pregnancy and the Development of Offspring. Biological Trace Element Research 2013, 156, https://doi.org/10.1007/s12011-013-9836-z.

32. Ojo, J.O.; Algamal, M; Leary, P. Disruption in Brain Phospholipid Content in a Humanized Tau Transgenic Model Following Repetitive Mild Traumatic Brain Injury. Frontiers Neuroscince 2018, 12, https://doi.org/10.3389/fnins.2018.00893.

33. Falone, S; Santini, S; Cordone, V. Extremely Low-Frequency Magnetic Fields and Redox-Responsive Pathways Linked to Cancer Drug Resistance: Insights from Co-Exposure-Based In Vitro Studies. Frontiers Public Health 2018, 6, https://doi.org/10.3389/fpubh.2018.00033.

34. Ross, SM. Combinated DC and ELF magnetic fields can alter cell proliferation. Bioelectromagnetics 1990,11, 27-36, https://doi.org/10.1002/bem.2250110105

35. Hoy, M.A. Insect Molecular Genetics An Introduction to Principles and Applications. Elsevier 2019, 705, https://doi.org/10.1016/c2017-0-02824-0.

36. de Bruyn, L.; de Jager, L. Effect of Long-Term Exposure to a Randomly Varied $50 \mathrm{~Hz}$ Power Frequency Magnetic Field on the Fertility of the Mouse Electromagnetic Biology and Medicine 2010, 29, 52-61, https://doi.org/10.3109/15368371003776659.

37. Miller, L.M.; Dumas, P. Chemical Imaging of Biological Tissue with Synchrotron Infrared Light. Biochimica et Biophysica Acta Biomembranes 2006, 1758, 846-57, https://doi.org/10.1016/j.bbamem.2006.04.010. 